\title{
Long term effects of smoking cessation in hospitalized schizophrenia patients
}

Masatoshi Miyauchi ${ }^{1,2^{*}}$ (D) Ikuko Kishida ${ }^{1}$, Akira Suda ${ }^{1}$, Yohko Shiraishi ${ }^{1,3}$, Mami Fujibayashi ${ }^{4}$, Masataka Taguri ${ }^{5}$, Chie Ishii $^{2}$, Norio Ishii ${ }^{2}$, Toshio Moritani ${ }^{6}$ and Yoshio Hirayasu ${ }^{1}$

\begin{abstract}
Background: The prevalence of smoking in patients with schizophrenia is higher than that in the general population and is an important medical issue. Short-term smoking cessation tends to worsen psychiatric symptoms in patients with schizophrenia but decreases sympathetic nervous system activity and improves plasma cholesterol levels in healthy people. Few studies have assessed the long-term effects of smoking cessation in patients with schizophrenia.
\end{abstract}

Methods: Subjects were 70 Japanese patients with schizophrenia (38 smokers, 32 non-smokers). We compared the following clinical parameters between the two groups at baseline (before smoking cessation) and in each group separately between baseline and at three years after smoking cessation: autonomic nervous system activity, plasma cholesterol levels, body weight, drug therapy, and Global Assessment of Functioning scores. We also compared the mean changes in clinical parameters throughout this study between the groups at both time points. Autonomic nervous system activity was assessed by power spectral analysis of heart rate variability.

Results: Parasympathetic nervous system activity and the doses of antiparkinsonian drugs in smokers were significantly higher than those in non-smokers at baseline. Smoking cessation was associated with significantly decreased sympathetic nervous system activity and decreased doses of antipsychotics and antiparkinsonian drugs at three years after smoking cessation. However, there was no significant difference in the mean change in clinical factors scores, except for Global Assessment of Functioning scores, between smokers and non-smokers at three years after smoking cessation.

Conclusions: Our results suggest that smoking reduces both autonomic nervous system activity and the effectiveness of drug therapy with antipsychotics and antiparkinsonian drugs in patients with schizophrenia, but that both factors could be ameliorated over the long term by smoking cessation. Taken together with the findings of previous studies, smoking cessation in patients with schizophrenia has many long-term positive physiological effects.

Keywords: Autonomic nervous system, Patient with schizophrenia, Smoking cessation, Antipsychotics, Antiparkinsonian drug, Cholesterol, Body mass index

\footnotetext{
* Correspondence: miyauchi-masatoshi@hotmail.co.jp

${ }^{1}$ Department of Psychiatry, Yokohama City University School of Medicine, 3-9

Fukuura, Kanazawa-ku, Yokohama, Kanagawa 236-0004, Japan

${ }^{2}$ Fujisawa Hospital, 383 Kotsuka, Fujisawa, Kanagawa 251-8530, Japan

Full list of author information is available at the end of the article
} 


\section{Background}

The prevalence of smoking in patients with schizophrenia is at least two to three times higher than that in the general population [1-3]. Patients with schizophrenia experience greater difficulties in smoking cessation compared with the general population [1-3]. Given the problems associated with smoking, this habit among patients with schizophrenia is of clinical significance. Smoking cessation has various positive physiological effects in healthy people, including lower serum cholesterol levels and decreased sympathetic nervous system activity [4-9]; increased sympathetic nervous activity increases the risk of cardiac disease, such as atrial and ventricular arrhythmias, cardiac arrest, and acute myocardial infarction [10-13]. However, as a negative physiological effect, a previous study reported that smoking cessation for one year resulted in weight gain [14].

Several studies have investigated the effects of smoking and smoking cessation in patients with schizophrenia. One study showed an association between smoking and high serum lipid profiles [15], and another found that smoking cessation for one year was associated with worsening of Global Assessment of Functioning (GAF) scores [16]. However, few studies have assessed the longterm effects of smoking cessation in these patients. The present study aimed to address this gap by investigating the long-term effects of smoking cessation in patients with schizophrenia, such as the effects on serum cholesterol levels, body weight, drug therapy, GAF scores, and autonomic nervous system (ANS) activity.

\section{Methods}

\section{Subjects}

This study involved 38 smokers ( 7 men, 31 women; 57.9 \pm 12.5 years) and 32 non-smokers ( 11 men, 21 women; $54.3 \pm 13.6$ years) with schizophrenia diagnosed according to the Diagnostic and Statistical Manual of Mental Disorders, fourth edition (DSM-IV), by a psychiatrist. They were all inpatients at a psychiatric hospital during the study period. Exclusion criteria were (1) onset of chronic medical conditions (e.g., diabetes, respiratory disease, cardiac disease, or ANS disorders) at any point during the study and (2) cardiovascular disease, arrhythmia, and long QT syndrome as determined in all patients by chest radiography and electrocardiography. Regular blood tests, chest radiography and electrocardiography were performed once a year and subjects were evaluated by a psychiatrist regularly throughout the study period. All 38 smokers were habitual smokers, with a Tobacco Dependence Screener (TDS) score of $>5$ points (mean score 5.71 $\pm 0.65)$. The TDS is a self-administered questionnaire for assessing nicotine dependence as defined by the 10th revision of the International Statistical Classification of Diseases and Related Health Problems and DSM-IV [17], the cutoff score for TDS is 5 points for diagnosis according to ICD-10 [17]. The 32 non-smokers had never smoked before. Based on the stipulation of the hospital's own smoke-free policy instituted in 2010, the 38 smokers quit smoking. After instituting the policy, we assessed the subjects over the subsequent three-year period. None of the 38 smokers received any medication or behavioral therapy for smoking cessation.

The clinical parameters studied at baseline (before smoking cessation in smokers) and at three years after smoking cessation were all related to the effects of smoking. These parameters included body mass index (BMI) and serum levels of albumin (ALB), blood sugar (BS), low-density lipoprotein cholesterol (LDL-C), total cholesterol (TC), triglycerides (TG), and high-density lipoprotein cholesterol (HDL-C). In addition, subjects were evaluated and assigned GAF scores according to DSM-IV as assessed by a psychiatrist at baseline and at three years after smoking cessation. We also investigated the use of antipsychotics, antiparkinsonian drugs, and anxiolytics, namely chlorpromazine, biperiden, and diazepam, respectively. The required therapeutic doses of these drugs were calculated for each patient according to the standard equivalent conversions [18].

The study protocol was approved by the Institutional Review Board of Seishinkai Fujisawa Hospital, and the study was performed in accordance with the Declaration of Helsinki. All patients provided written informed consent after receiving detailed information regarding the study.

\section{R-R interval power spectral analysis}

ANS activity was quantified using heart rate variability (HRV) power spectral analysis. The technique employed in the present investigation for detecting the three major spectral components of HRV has been previously applied under diverse psychophysiological conditions in basic medical science and clinical research. The validity, reliability, and practicability of this technique have been well documented [19-21]. HRV power spectral analysis decomposes a series of sequential $R-R$ intervals obtained from electrocardiography (ECG) for $5 \mathrm{~min}$ into a sum of sinusoidal functions of different amplitudes and frequencies by the Fast Fourier transform algorithm [22-27]. The spectral powers in the frequency domain were quantified by integrating the area under the curve for the following respective bandwidth measurements: the low-frequency (LF; $0.03-0.15 \mathrm{~Hz}$ ) component of HRV representing both sympathetic and parasympathetic activity; the high-frequency (HF; 0.15-0.4 Hz) component associated almost entirely with parasympathetic nerve activity; and total power (TP; 0.03-0.4 Hz) representing overall cardiac ANS activity [24-26]. 


\section{Statistical analysis}

The clinical parameters (HRV; BMI; plasma levels of ALB, TP, BS, LDL-C, TC, TG, and HDL-C; doses of antipsychotics, antiparkinsonian drugs, and anxiolytic drugs; and GAF scores) were compared between smokers and non-smokers at baseline using Student's unpaired $t$-test, and in each group separately between baseline and at three years after smoking cessation using Wilcoxon's signed-rank test. Additionally, to evaluate the effects of smoking cessation, mean changes in the clinical parameters were compared between the two groups at both time points using the unpaired $t$-test. Total power, LF, and HF data were logarithmically transformed using Log10 to obtain normally distributed data. All statistical analyses were performed with SPSS for Windows Version 21.0 (IBM Corp., Armonk, NY). A $p$ value of $<0.05$ was considered statistically significant.

\section{Results}

Table 1 shows the baseline demographic and clinical parameters for all subjects. The 38 smokers comprised 7 men and 31 women, with a mean age ( \pm standard deviation) of $58.5 \pm 2.3$ years; the 32 non-smokers comprised 11 men and 21 women with a mean age of $54.3 \pm$ 13.6 years. Mean duration of illness was $33.2 \pm 14.5$ years and $32.7 \pm 13.5$ years, respectively. Compared with nonsmokers, smokers had a significantly higher HF component and significantly higher doses of antiparkinsonian drugs (both $p<0.05$ )

Table 2 shows the clinical parameters at baseline and at three years after smoking cessation in both groups as well as the mean changes at both time points. At three years after smoking cessation compared with at baseline, smokers had a significantly lower LF component and significantly decreased doses of antipsychotics and antiparkinsonian drugs (both $p<0.05$ ). Most subjects were receiving $>1$ antipsychotic. BMI was significantly decreased and serum HDL-C was significantly increased in both groups (both $p<0.05$ ). Among the mean changes in clinical parameters analyzed, only GAF score was significantly different between smokers and non-smokers, showing significant worsening in smokers $(p<0.05)$.

\section{Discussion}

\section{Comparison of clinical parameters between smokers and non-smokers at baseline}

Our results showed that smokers had a higher HF component than non-smokers, suggesting increased parasympathetic nervous system activity in smokers with schizophrenia. This finding differs from that in healthy subjects, in which smokers had higher sympathetic nervous system activity than non-smokers [4-6], and it supports the previous findings of abnormal autonomic nervous system activity in patients with schizophrenia
Table 1 Clinical parameters between smokers and non-smokers at baseline (before cessation of smoking)

\begin{tabular}{|c|c|c|c|}
\hline & Smokers & Non-smokers & $P$ value \\
\hline Number & 38 & 32 & \\
\hline Sex & $7 / 31$ & $11 / 21$ & \\
\hline Age (years) & $57.9 \pm 12.5$ & $54.3 \pm 13.6$ & 0.241 \\
\hline Duration of illness & $33.2 \pm 14.5$ & $32.7 \pm 13.5$ & 0.876 \\
\hline In total ${ }^{1}$ & $4.5 \pm 1.2$ & $3.9 \pm 1.2$ & 0.071 \\
\hline $\ln \mathrm{LF}^{1}$ & $3.8 \pm 1.3$ & $3.2 \pm 1.4$ & 0.163 \\
\hline In $\mathrm{HF}^{1}$ & $3.8 \pm 1.2$ & $3.1 \pm 1.3$ & $0.016^{*}$ \\
\hline BMI $\left(\mathrm{kg} / \mathrm{m}^{2}\right)$ & $22.7 \pm 4.0$ & $21.7 \pm 4.0$ & 0.276 \\
\hline ALB $(g / d L)$ & $4.0 \pm 0.3$ & $4.0 \pm 0.4$ & 0.204 \\
\hline BS (mg/dL) & $89.7 \pm 17.4$ & $87.5 \pm 15.5$ & 0.655 \\
\hline LDL-C (mg/dL) & $119.0 \pm 33.2$ & $108.4 \pm 29.6$ & 0.163 \\
\hline TC (mg/dL) & $200.4 \pm 42.2$ & $183.9 \pm 32.0$ & 0.065 \\
\hline TG (mg/dL) & $118.9 \pm 91.1$ & $86.0 \pm 43.2$ & 0.073 \\
\hline $\mathrm{HDL}-\mathrm{C}(\mathrm{mg} / \mathrm{dL})$ & $60.4 \pm 16.6$ & $55.9 \pm 13.2$ & 0.222 \\
\hline CPZeq ${ }^{* 2}(\mathrm{mg})$ & $1229 \pm 750$ & $1162 \pm 792$ & 0.717 \\
\hline BPDeq $^{* 2}(\mathrm{mg})$ & $2.9 \pm 1.9$ & $1.6 \pm 1.6$ & $0.003^{*}$ \\
\hline DZPeq $^{* 2}(\mathrm{mg})$ & $10.1 \pm 6.9$ & $9.3 \pm 7.4$ & 0.653 \\
\hline GAF score & $28.9 \pm 3.1$ & $29.2 \pm 2.2$ & 0.682 \\
\hline
\end{tabular}

Data are presented as means \pm standard deviation

* Significant difference $(p<0.05)$

'Logarithmic conversion was performed

${ }^{2}$ Daily dose of antipsychotic was converted to the approximate respective equivalent dose

LF Low Frequency, HF High frequency, BMI Body mass index, ALB Serum albumin, $B S$ Blood sugar, LDL-C Low-density lipoprotein cholesterol, TC Total cholesterol, TG Triglycerides, HDL-C High-density lipoprotein cholesterol, CPZeq Equivalent conversions of chlorpromazine, BDPeq Equivalent conversions of biperiden, DZP Equivalent conversions of diazepam, GAF Global Assessment of Functioning

compared with healthy people $[28,29]$. The required doses of antiparkinsonian drugs at baseline were significantly higher in smokers than in non-smokers. We suggest that this finding is accounted for by higher required doses of antipsychotic drugs in smokers compared with non-smokers.

\section{Clinical parameters at baseline and at three years after smoking cessation}

Our results demonstrate that long-term smoking cessation in smokers with schizophrenia was associated with a decreased LF component, which might indicate reduced sympathetic nervous system activity resulting from smoking cessation. Smoking stimulates nicotinic acetylcholine receptors in the central nervous system and increases inflammation and oxidative stress in the lungs, leading to increased sympathetic activity via activation of afferent neurons in the lungs [4]. Thus, our finding suggesting that smoking cessation improved sympathetic nervous system activity seems reasonable. In contrast, the HF component showed no significant 
Table 2 Changes in clinical parameters between baseline and three years later in smokers and in non-smokers

\begin{tabular}{|c|c|c|c|c|c|c|c|c|c|}
\hline & \multicolumn{4}{|l|}{$\begin{array}{l}\text { Smokers } \\
n=38\end{array}$} & \multicolumn{4}{|l|}{$\begin{array}{l}\text { Non-smokers } \\
n=32\end{array}$} & \multirow[b]{2}{*}{ Comparison } \\
\hline & Baseline & Three years & Changes & $p$ & Baseline & Three years & Changes & $P$ & \\
\hline In total ${ }^{1}$ & $4.5 \pm 1.2$ & $4.0 \pm 1.7$ & $-0.50 \pm 1.57$ & 0.061 & $3.9 \pm 1.2$ & $3.9 \pm 1.4$ & $-0.11 \pm 0.95$ & 0.681 & 0.218 \\
\hline $\ln L F^{1}$ & $3.8 \pm 1.3$ & $3.0 \pm 1.8$ & $-0.79 \pm 1.73$ & $0.049^{*}$ & $3.2 \pm 1.4$ & $3.2 \pm 1.5$ & $-0.09 \pm 0.96$ & 0.715 & 0.133 \\
\hline In HF & $3.8 \pm 1.2$ & $3.4 \pm 1.7$ & $-0.44 \pm 1.67$ & 0.136 & $3.1 \pm 1.3$ & $3.1 \pm 1.5$ & $-0.85 \pm 1.17$ & 0.915 & 0.304 \\
\hline BMI $\left(\mathrm{kg} / \mathrm{m}^{2}\right)$ & $22.7 \pm 4.0$ & $21.1 \pm 3.6$ & $-1.56 \pm 3.64$ & $0.020^{*}$ & $21.7 \pm 4.0$ & $20.8 \pm 3.9$ & $-0.92 \pm 2.17$ & $0.022^{*}$ & 0.373 \\
\hline ALB (g/dL) & $4.0 \pm 0.3$ & $3.9 \pm 0.4$ & $-0.03 \pm 0.38$ & 0.564 & $4.0 \pm 0.4$ & $3.9 \pm 0.4$ & $-0.15 \pm 0.46$ & 0.076 & 0.240 \\
\hline $\mathrm{BS}(\mathrm{mg} / \mathrm{dL})$ & $89.7 \pm 17.4$ & $87.6 \pm 14.6$ & $-1.7 \pm 18.6$ & 0.568 & $87.5 \pm 15.5$ & $87.7 \pm 14.1$ & $0.16 \pm 16.2$ & 0.957 & 0.654 \\
\hline $\mathrm{LDL}-\mathrm{C}(\mathrm{mg} / \mathrm{dL})$ & $119.0 \pm 33.2$ & $115.6 \pm 36.2$ & $-2.3 \pm 39.0$ & 0.717 & $108.4 \pm 29.6$ & $110.2 \pm 27.3$ & $1.7 \pm 29.2$ & 0.740 & 0.631 \\
\hline $\mathrm{TC}(\mathrm{mg} / \mathrm{dL})$ & $200.4 \pm 42.2$ & $190.4 \pm 42.2$ & $-10.4 \pm 52.1$ & 0.291 & $183.9 \pm 32.0$ & $186.3 \pm 35.1$ & $2.4 \pm 27.2$ & 0.620 & 0.421 \\
\hline $\mathrm{TG}(\mathrm{mg} / \mathrm{dL})$ & $118.9 \pm 91.1$ & $100.0 \pm 48.0$ & $-18.9 \pm 93.1$ & 0.212 & $86.0 \pm 43.2$ & $96.1 \pm 40.8$ & $10.2 \pm 46.2$ & 0.223 & 0.096 \\
\hline $\mathrm{HDL}-\mathrm{C}(\mathrm{mg} / \mathrm{dL})$ & $60.4 \pm 16.6$ & $70.3 \pm 18.0$ & $9.92 \pm 18.2$ & $0.002^{*}$ & $55.9 \pm 13.2$ & $64.0 \pm 18.9$ & $8.1 \pm 13.5$ & $0.002^{*}$ & 0.635 \\
\hline CPZeq ${ }^{2}(\mathrm{mg})$ & $1229 \pm 750$ & $1008 \pm 646$ & $-221 \pm 576$ & $0.031^{*}$ & $1162 \pm 792$ & $1075 \pm 810$ & $-86.7 \pm 489$ & 0.323 & 0.302 \\
\hline $\mathrm{BPDeq}^{2}(\mathrm{mg})$ & $2.9 \pm 1.9$ & $2.3 \pm 1.7$ & $-0.63 \pm 1.7$ & $0.018^{*}$ & $1.6 \pm 1.6$ & $1.7 \pm 1.7$ & $0.15 \pm 1.8$ & 0.623 & 0.061 \\
\hline $\mathrm{DZPeq}^{2}(\mathrm{mg})$ & $10.1 \pm 6.9$ & $8.6 \pm 6.9$ & $-1.5 \pm 6.4$ & 0.190 & $9.3 \pm 7.4$ & $9.1 \pm 7.4$ & $-0.12 \pm 6.6$ & 0.914 & 0.379 \\
\hline GAF score & $28.9 \pm 3.1$ & $27.9 \pm 4.1$ & $-1.1 \pm 3.1$ & 0.444 & $29.2 \pm 2.2$ & $29.4 \pm 2.1$ & $0.15 \pm 1.5$ & 0.572 & $0.040^{*}$ \\
\hline
\end{tabular}

Data are presented as means \pm standard deviation

*Significant difference $p<0.05$

${ }^{1}$ Logarithmic conversion was performed

${ }^{2}$ The daily dose of antipsychotics was converted to the approximate respective equivalent dose

$L F$ Low frequency, HF High frequency, BMI Body mass index, ALB Albumin, BS Blood sugar, LDL-C Serum low-density lipoprotein cholesterol, TC Total cholesterol, TG Triglycerides, HDL-C High-density lipoprotein cholesterol, CPZeq Equivalent conversions of chlorpromazine, $B D P e q$ Equivalent conversions of biperiden, DZP

Equivalent conversions of diazepam, GAF Global Assessment of Functioning

change after long-term smoking cessation in our patient population, which is inconsistent with the findings of previous studies involving healthy subjects [4, 30, 31]. Given that patients with schizophrenia have much lower parasympathetic nervous system activity than healthy people $[28,29]$, our findings seem to be influenced more by this much lower activity than by smoking.

In this study, the required doses of antipsychotics and antiparkinsonian drugs of the smokers were significantly decreased after smoking cessation, but the doses of anxiolytic drugs were not affected. Because smoking increases the activity of cytochrome P450 isoenzyme 1A2 (CYP1A2) and UDP-glucuronosyltransferases (UGT), which are responsible for drug metabolism, the metabolism of antipsychotics is consequently influenced by smoking [32]. Smoking cessation therefore results in decreased metabolism of antipsychotics via CYP1A2 and UGT and thus in the required dose of antipsychotics. This is supported by the finding that, after smoking cessation, dopamine levels were increased following a decrease in the stimulation of nicotinic acetylcholine receptors [33]. Our results also suggest that smoking cessation in the long term decreased the required dose of antiparkinsonian drugs, as a consequence of taking lower doses of antipsychotics after smoking cessation.

In this study, smoking cessation in inpatients with schizophrenia lowered serum HDL-C, similar to findings in healthy individuals [7, 9]. Moreover, we found that BMI was decreased after smoking cessation in these inpatients, although this finding conflicts with that of a meta-analysis reporting that people experience an increase in body weight after smoking cessation because of improved appetite [14]. This discrepancy might be because our subjects were inpatients and received hospital meals monitored by a nutritionist every day throughout this study. In smokers with schizophrenia, some antipsychotics cause substantial weight gain and therefore the reduced required dose of antipsychotics that we observed may have resulted in the decreased BMI observed after smoking cessation. Moreover, because patients with schizophrenia usually gain weight after smoking cessation, we educated all subjects to avoid weight gain. Further research is needed to confirm this speculation. Our results showed that both smokers and non-smokers had lower HDL-C and BMI in the long term and this may have been influenced by factors other than smoking cessation.

\section{Mean changes in clinical parameters at three years among smokers and non-smokers}

Our results showed a significant difference in GAF scores between smokers and non-smokers. A previous study revealed that smoking cessation worsened psychotic symptoms and increased the propensity for violence 
in psychiatric patients [34]. Moreover, another study reported worsened GAF scores at one year after smoking cessation [16], which is consistent with our long-term assessment results [16]. However, we found no significant difference in clinical factors that could explain the difference in GAF score. Further study is needed to assess the effects on psychotic symptoms in the long term.

\section{Limitations of the study}

Our study has some limitations. First, it was conducted at a single institution and the number of subjects was relatively small. This does not adequately reflect significant differences in the mean changes in clinical parameters between baseline and at three years among smokers and non-smokers. Second, we did not investigate patients with schizophrenia who continued to smoke and we did not consider the effect of sex on cholesterol levels and ANS activity. Third, our assessment of whether subjects smoked or not was subjective, not objective. Fourth, we did not analyze the long-term effects of smoking cessation in the short term and whether smoking cessation affected psychiatric symptoms such as indicated by the Positive and Negative Syndrome Scale (PANSS). Fifth, we did not determine the serum concentrations of the antipsychotics and did not consider the metabolism of individual antipsychotics directly influenced by smoking, including olanzapine and haloperidol. Sixth, we did not take multiple testing into account because this was exploratory research. Finally, most of our subjects were patients with schizophrenia who had been hospitalized for a long time and these results do not therefore apply to all patients with schizophrenia. Further study with a larger sample size and other subjects such as outpatients with schizophrenia is needed to verify our results.

\section{Conclusions}

Our findings suggest that long-term effects of smoking cessation among inpatients with schizophrenia were not only associated with decreased required doses of antipsychotics and antiparkinsonian drugs but also with improved ANS activity. Further studies are needed to clarify whether smoking cessation leads to long-term changes in the health of patients with schizophrenia.

\footnotetext{
Abbreviations

ALB: Serum albumin; ANS: Autonomic nervous system (ANS); BMI: Body mass index; BPDeq: Equivalent conversions of biperiden; BS: Blood sugar; CPZeq: Equivalent conversions of chlorpromazine; DZPeq: Equivalent conversions of diazepam; GAF: Global Assessment of Functioning; HDLC: High-density lipoprotein cholesterol; HF: High frequency; HRV: Heart rate variability; LDL-C: Low-density lipoprotein cholesterol; LF: Low frequency; TC: Total cholesterol; TG: Triglycerides
}

Funding

This study received no specific funding.

\section{Availability of data and materials}

The datasets used and analysed during the current study available from the corresponding author on reasonable request.

\section{Authors' contributions}

MM was involved in the design of the study, collection and analysis of data, and writing the first draft of the manuscript. IK was involved in the design of the study, collection and analysis of data, supervision of statistical analyses, and revision of the manuscript. AS was involved in analysis of data, supervision of statistical analyses, and revision of the manuscript. YS was involved in collection and analysis of data. MF was involved in analysis of data, particularly assessment of heart rate variability by power spectral analysis. MT was involved in analysis of data. CI was involved in the design of the study and collection and analysis of data. NI was involved in the design of the study, collection and analysis of data, and supervision of statistical analyses. TM was involved in analysis of data, particularly assessment of heart rate variability by power spectral analysis. YH was involved in the design of the study, collection and analysis of data, supervision of statistical analyses, and revision of the manuscript. All authors read and approved the final manuscript.

\section{Competing interests}

The authors declare that they have no competing interests.

\section{Consent for publication}

Not applicable.

\section{Ethics approval and consent to participate}

The study protocol was approved by the Institutional Review Board of Seishinkai Fujisawa Hospital, and the study was performed in accordance with the Declaration of Helsinki. All patients provided written informed consent after receiving detailed information regarding the study.

\section{Author details}

${ }^{1}$ Department of Psychiatry, Yokohama City University School of Medicine, 3-9 Fukuura, Kanazawa-ku, Yokohama, Kanagawa 236-0004, Japan. ²Fujisawa Hospital, 383 Kotsuka, Fujisawa, Kanagawa 251-8530, Japan. ${ }^{3}$ Clinical Laboratory Department, Yokohama City University Hospital, 3-9 Fukuura, Kanazawa-ku, Yokohama, Kanagawa 236-0004, Japan. ${ }^{4}$ The Division of Physical and Health Education, Setsunan University, 17-8 Ikedanakamachi, Neyagawa, Osaka 572-8508, Japan. ${ }^{5}$ Department of Biostatistics, Yokohama City University School of Medicine, 3-9 Fukuura, Kanazawa-ku, Yokohama, Kanagawa 236-0004, Japan. ${ }^{6}$ Faculty of General Education, Kyoto Sangyo University, Kamo-motoyama, Kita-Ku, Kyoto 606-8555, Japan.

Received: 19 October 2016 Accepted: 2 March 2017

Published online: 07 March 2017

\section{References}

1. Dalack GW, Healy DJ, Meador-Woodruff JH. Nicotine dependence in schizophrenia: clinical phenomena and laboratory findings. Am J Psychiatry. 1988;155:1490-501. doi:10.1176/ajp.155.11.1490.

2. de Leon J, Diaz FJ. A meta-analysis of worldwide studies demonstrates an association between schizophrenia and tobacco smoking behaviors. Schizophr Res. 2005;76:135-57. doi:10.1016/j.schres.2005.02.010.

3. Kelly C, McCreadie R. Cigarette smoking and schizophrenia. Adv Psychiatr Treat. 2000;6:327-31. doi:10.1192/apt.6.5.327.

4. Middlekauff HR, Park J, Moheimani RS. Adverse effects of cigarette and noncigarette smoke exposure on the autonomic nervous system: mechanisms and implications for cardiovascular risk. J Am Coll Cardiol. 2014;64:1740-50. doi:10.1016/j.jacc.2014.06.1201.

5. Dians PC, Koutedakis Y, Flouris AD. Effects of active and passive tobacco cigarette smoking on heart rate variability. Int J Cardiolo. 2013;163:109-15. doi:10.1016/j.ijcard.2011.10.140.

6. Barutchu I, Esen AM, Kaya D, Turkmen M, Karakaya O, Melek M, et al. Cigarette smoking and heart rate variability; dynamic influence of parasympathetic maneuvers. Ann Noninvasive Electrocardiol. 2005;10:324-9. doi:10.1111/j.1542-474X.2005.00636.x. 
7. Maeda K, Noguchi Y, Fukui T. The effects of cessation from cigarette smoking on the lipid and lipoprotein profiles: a meta-analysis. Prev Med. 2003;37:283-90

8. Mjøs OD. Lipid effects of smoking. Am Heart J. 1988;115:272-5.

9. Takata K, Imaizumi S, Kawachi E, Suematsu Y, Shimizu T, Abe S, et al. Impact of cigarette smoking cessation on high-density lipoprotein functionality. Circ J. 2014;78:2955-62. doi:10.1253/circj.CJ-14-0638.

10. Sandhu RK, Jimenez MC, Chiuve SE, et al. Smoking, smoking cessation, and risk of sudden cardiac death in women. Circ Arrhtthim Electrophysiol. 2012; 5:1091-7.

11. Smith RC, Warner-Cohen J, Matute M, Butler E, Kelly E, Vaidhyanathaswamy S, Khan A. Effects of nicotine nasal spray on cognitive function in schizophrenia. Neuropsychopharmacology. 2006;31:637-43.

12. Stand JE, Nyback H. Tobocco use in schizophrenia: a study of cotinine concentrations in the saliva of patients and controls. Eur Psychiatry. 2005;20:50-4.

13. Stein PK, Rottman JN, Kliger RE. Effects of 21 mg transdermal nicotine patches and smoking cessation on heart rate variability. Am J Cardiol. 1996;77:701-5.

14. Aubin HJ, Ferley A, Lycett D, Lahmerk P, Aveyard P. Weight gain in smokers after quitting cigarettes: meta-analysis. BMJ. 2012;345:e4439. doi:10.1136/ bmj.e4439.

15. An HM, Tan YL, Tan SP, Shi J, Wang ZR, Yang FD, et al. Neurosci. Bull. 2016; 32:383-8. doi:10.1007/s12264-016-0022-0.

16. Cole ML, Triqoboff E, Demler TL, Opler LA. Impact of smoking cessation on psychiatric inpatients treated with clozapine or olanzapine. J Psychiatr Pract. 2010;16:1675-81. doi:10.1097/01.pra.0000369968.80155.3f.

17. Kawakami N, Takatsuka N, Inaba S, Shimizu H. Development of a screening questionnaire for tobacco/nicotine dependence according to ICD-10, DSMIII-R, and DSM-IV. Addict Behav. 1999;24:155-66.

18. Inada T, Inagaki A. Psychotropic dose equivalence in Japan. Psychiatry Clin Neurosci. 2015:69:440-7. doi:10.1111/pen.12275.

19. Moritani T, Kimura T, Hamada T, Nagai N. Electrophysiology and kinesiology for health and disease. J Electromyogr Kinesiol. 2005;15:240-55. doi:10.1016/ j.jelekin.2005.01.001.

20. Matsumoto T, Miyawaki T, Ue H, Kanda T, Zenji C, Moritani T. Autonomic responsiveness to acute cold exposure in obese and non-obese young women. Int J Obes Relat Metab Disord. 1999;23:793-800. doi:10.1038/sj.ijo. 0800928.

21. Matsumoto T, Ushiroyama T, Morimura M, Moritani T, Hayashi T, Suzuki T, et al. Autonomic nervous system activity in the late luteal phase of eumenorrheic women with premenstrual symptomatology. J Psychosom Obstet Gynaecol. 2006;27:131-9. doi:10.1080/01674820500490218.

22. Fujibayashi M, Matsumoto T, Kishida I, Kimura T, Ishii C, Ishii N, et al. Autonomic nervous system activity and psychiatric severity in schizophrenia. Psychiatry Clin Neurosci. 2009;63:538-45. doi:10.1111/j.1440-1819.2009.01983.x.

23. Kimura T, Matsumoto T, Akiyoshi M, Owa Y, Miyasaka N, Aso T, et al. Body fat and blood lipids in postmenopausal women are related to resting autonomic nervous system activity. Eur J Appl Physiol. 2006;97:542-7. doi:10.1007/s00421-006-0207-8.

24. Akselrod S, Gordon D, Ubel FA, Shannon DC, Berger AC, Cohen RJ. Power spectrum analysis of heart rate fluctuation: a quantitative probe of beat-tobeat cardiovascular control. Science. 1981;213:220-2. doi:10.1126/science. 6166045.

25. Pagani M, Lombardi F, Guzzetti S, Rimoldi O, Furlan R, Pizzinelli P, et al. Power spectral analysis of heart rate and arterial pressure variabilities as a marker of sympatho-vagal interaction in man and conscious dog. Circ Res. 1986:59:178-93. doi:10.1161/01.RES.59.2.178.

26. Pomeranz B, Macaulay RJ, Caudill MA, Kutz I, Adam D, Gordon D, et al. Assessment of autonomic function in humans by heart rate spectral analysis. Am J Physiol. 1985;248:H151-3.

27. Rompelman O, Coenen A, Kitney R. Measurement of heart-rate variability: part 1-comparative study of heart-rate variability analysis methods. Med Biol Eng Comput. 1977;15:232-9. doi:10.1007/BF02441043.

28. Clamor A, Lincoln TM, Thayer JF, Koeniq J. Resting vagal activity in schizophrenia: meta-analysis of heart rate variability as a potential endophenotype. Br J Psychiatry. 2016;208:9-16. doi:10.1192/bjp.bp.114. 160762.

29. Montaquila JM, Trachik BJ, Bedwell JS. Heart rate variability and vagal tone in schizophrenia: A review. J Psychiatr Res. 2015;69:57-66. doi:10.1016/j. jpsychires.2015.07.025.
30. Minami J, Ishimitsu T, Matsuoka H. Effects of smoking cessation on blood pressure and heart rate variability. Hypertension. 1999:33:586-90.

31. Yotsukura M, Koide Y, Fujii K, Tomono Y, Katayama A, Ando H, et al. Heart rate variability during the first month of smoking cessation. Am Heart J. 1988;135:1004-9.

32. Spina $\mathrm{E}$, de Leon J. Metabolic drug interactions with newer antipsychotics: a comparative review. Basic Clin Pharmacol Toxicol. 2007;100:4-22. doi:10.1111/j.1742-7843.2007.00017.x.

33. Yang YK, Nelson L, Kamaraju L, Wilson W, McEvoy JP. Nicotine decreases bradykinesia-rigidity in Haloperidol-treated patients with schizophrenia. Neuropsychopharmacology. 2002;27:684-6. doi:10.1016/S0893133X(02)00325-1.

34. Freedman R. Exacerbation of schizophrenia by varenicline. Am J Psychiatry. 2007;164:1269. doi:10.1176/appi.ajp.2007.07020326.

\section{Submit your next manuscript to BioMed Central and we will help you at every step:}

- We accept pre-submission inquiries

- Our selector tool helps you to find the most relevant journal

- We provide round the clock customer support

- Convenient online submission

- Thorough peer review

- Inclusion in PubMed and all major indexing services

- Maximum visibility for your research

Submit your manuscript at www.biomedcentral.com/submit 